\title{
Heavy quark transport in a viscous semi-QGP
}

\author{
Balbeer Singh ${ }^{1,2}$ and Hiranmaya Mishra $\odot^{1}$ \\ ${ }^{1}$ Theory Division, Physical Research Laboratory, Navrangpura, Ahmedabad 380 009, India \\ ${ }^{2}$ Indian Institute of Technology Gandhinagar, Gandhinagar 382 355, Gujarat, India
}

(Received 15 November 2019; accepted 29 February 2020; published 18 March 2020)

\begin{abstract}
We study the effect of shear and bulk viscosities on the heavy quark transport coefficient within the matrix model of semi-QGP. Dissipative effects are incorporated through the first-order viscous correction in the quark/antiquark and gluon distribution function. It is observed that while the shear viscosity effects reduce the drag of heavy quark the bulk viscosity effects increase the drag and the diffusion coefficients of heavy quark. For finite values of $\eta / s$ and $\xi / s$, the Polyakov loop further decreases the drag coefficient and enhances the momentum diffusion coefficient as compared to perturbative QCD. It is also observed that with increases in $\eta / s$ and $\xi / s$ spatial diffusion coefficient decreases.
\end{abstract}

DOI: 10.1103/PhysRevD.101.054027

\section{INTRODUCTION}

The aim of heavy ion collision (HIC) experiments is to characterize the properties of the deconfined state of matter namely the quark-gluon plasma (QGP) which is being created in these collisions. In order to characterize the QGP, well-calibrated probes are required. In this context, the transport properties of heavy quark (HQ); especially charm and bottom; in the bulk of light thermal partons is considered as one of the promising probes. The interaction of heavy quarks with the thermal partons in the QGP medium reflects in the transverse momentum $\left(p_{T}\right)$ spectra of open heavy flavor (HF) meson such as $D$-meson for charm quark, and in the elliptic flow $v_{2}$ of open meson for a noncentral collision [1-7].

Heavy quarks are produced in the initial stages of the collisions during the hard scatterings governed by perturbative quantum chromodynamics (pQCD) mostly through gluon fusion [8-10]. Because of the large mass of HQ as compared to the temperature ranges accessible in the Relativistic Heavy Ion Collider (RHIC) and the Large Hadron Collider (LHC) energies, the thermal production of $\mathrm{HQ}$ is negligible. Hence, once produced in the hard collisions, HQ propagates throughout the space-time evolution of the medium and interact with the light thermal partons of the bulk medium. The resulting effect of the interaction of HQ with the bulk medium modifies the spectra of HF hadrons. The interaction of HQ with the bulk medium is described by the scattering of HQ with the light

Published by the American Physical Society under the terms of the Creative Commons Attribution 4.0 International license. Further distribution of this work must maintain attribution to the author(s) and the published article's title, journal citation, and DOI. Funded by SCOAP. thermal partons of the medium. At low momenta, the dominant contribution to the HQ scattering off of light quark and gluon in the thermal medium arises from the elastic scatterings and can be described by the diffusion process akin to Brownian motion. In addition, the thermalization of HQ in the bulk medium is also slowed down due to its large mass. Hence, the transport of nonequilibrated HQ in the thermalized medium of light quark and gluons yield valuable information about the medium throughout its propagation. In particular, the low momentum interaction of HQ with the bulk medium is characterized by the spatial diffusion coefficient.

Perturbative QCD based calculations for HQ transport coefficients namely the drag and the diffusion cannot explain the observed suppression and collective flow [11] so it is required to include the possible nonperturbative effects. There have been various efforts to incorporate nonperturbative effects using various phenomenological models such as the T-matrix model $[12,13]$, the quasiparticle model [14-16], and the resonance model [17] for estimating the HQ transport coefficients. The most reliable results for the HQ spatial diffusion comes from the first principle lattice simulations [18-20]. Indeed, a smaller value of the spatial diffusion coefficient as compared to the perturbative QCD is predicted by the lattice simulations which is essential to explain observed $R_{A A}$ and $v_{2}$. In Ref. [21], HQ transport coefficients (the drag and the momentum diffusion) are evaluated in the T-matrix approach including nonperturbative effects by employing the potential interaction of heavy-light quark extracted from lattice QCD simulations. A good agreement with the observed $R_{A A}$ and collective flow $v_{2}$ of this calculation suggests existence of the strongly interacting nature of QGP. Recently, based on a Polyakov loop model, heavy quark drag and the diffusion coefficients have been 
computed for the charm quark in Ref. [22]. The estimation of the drag coefficient here is observed being rather somewhat flat with temperature while the diffusion coefficient exhibited a strong temperature dependence similar to the results obtained in Refs. [12,21]. The consistency in the results suggest that there could be some model independent correlations between the results obtained within the Polyakov loop and other nonperturbative models from a different perspective.

On the other hand, it may also be mentioned here that QGP formed in HICs, behaves like almost an ideal fluid with a very small value for the ratio of shear viscosity to entropy density $\eta / s$. Evidence for such a small $\eta / s$ is provided by the large elliptic flow data that requires $\eta / s \sim$ $0.08-0.2$ [23-26]. Viscous coefficients in the QGP as well as in the hadronic medium have been studied in Refs. [2729]. In these studies it was found that the dominant contribution of dissipation in both the QGP and the hadronic medium arises from shear viscosity. However, bulk viscosity is equally important and may dominates near transition temperature i.e., $\xi / s \sim 1$ [30] and can significantly affect hadrons $p_{T}$ spectra and elliptic flow $v_{2}$ [31]. Viscous corrections have also been studied for dilepton production in QGP [32,33], photon production [34], damping rate of heavy quark [35], heavy quark radiative energy loss [36-38], event-plane correlations [39,40] etc. Effect of shear and bulk viscosities on the HQ drag and diffusion coefficients have been studied in Ref. [41] using a fugacity model. In the present study, we intend to include the viscous corrections (both shear and bulk) along with a nontrivial Polyakov loop background that is used to describe the "semi-QGP" within a matrix model. We find that in the perturbative limit our results are consistent with the previous results, however, with the inclusion of the Polyakov loop $(\phi)$, at low temperature our results are different from that of Ref. [41]. In this work, we include the viscous corrections (both shear and bulk) in the single particle distribution functions of quark and gluon to estimate the viscous effects on the HQ transport coefficients. We estimate this using Fokker-Planck equation and use the matrix model of semi QGP to evaluate the relevant scattering amplitudes. The single particle distribution function [see Eqs. (43) and (44)] is modified using second moment ansatz. In Ref. [36] it was shown that viscous effects induce a larger energy loss of HQ. So one may expect that viscous corrections may be important and significantly affect the transport properties of HQ in the bulk medium. However, we find that for small shear and bulk viscosities, the dissipative effects on the drag and the diffusion coefficients are somewhat weak.

We organize this work as follows: An introduction on the formalism for evaluation of HQ drag and diffusion coefficients within the matrix model of semi-QGP is discussed in Sec. II which is followed by the discussion on semi QGP in Sec. III. In this section, we also discuss some salient features of the matrix model. In Sec. IV, an ansatz for the first order viscous correction on quark/gluon distribution is discussed. In Sec. V, we discuss the interaction of HQ with the light thermal parton and present matrix element squared for Coulomb and Compton scatterings within the matrix model. These matrix element squared are used to evaluate the drag and diffusion coefficients. Finally, in Sec. VI we discuss the viscous effects on HQ quark transport and present the numerical results for the drag and the diffusion coefficient for constant values of $\eta / s$ and $\xi / s$. Finally, we summarize and give an outlook of the present work in Sec. VII.

\section{FORMALISM}

The Brownian motion of HF particles can be described by the Fokker-Planck equation where the interactions of heavy quark with the bulk of light quarks and gluons are encoded in the transport coefficient. Assuming that HF quark of momentum $\boldsymbol{p}$ is traveling in a medium of light quark and gluon, the Boltzmann equation for phase-space distribution $f_{Q}$ of heavy quark can be written as [42]

$$
\left[\frac{\partial}{\partial t}+\frac{\boldsymbol{p}}{E_{p}} \frac{\partial}{\partial \boldsymbol{x}}+\boldsymbol{F} \frac{\partial}{\partial \boldsymbol{p}}\right] f_{Q}(\boldsymbol{p}, \boldsymbol{x}, t)=C\left[f_{Q}\right],
$$

where $\boldsymbol{F}$ is the force due to external mean-field such as chromo electric or magnetic fields present in the initial stages of the heavy ion-collision, $E_{p}=\sqrt{m_{Q}^{2}+\boldsymbol{p}^{2}}$ is the energy of heavy quark with mass $m_{Q}$ and $C\left[f_{Q}\right]$ is the collision integral. Neglecting the mean-field effects, Eq. (1) reduces to

$$
\frac{\partial}{\partial t} f_{Q}(\boldsymbol{p}, t)=C\left[f_{Q}\right]
$$

On the right-hand side of Eq. (2), collision integral in terms of collision rate which change the momentum of HF quark from $\boldsymbol{p}$ to $\boldsymbol{p}-\boldsymbol{k}$ is written as

$$
C\left[f_{Q}\right]=\int d^{3} k\left[w(\boldsymbol{p}+\boldsymbol{k}, \boldsymbol{k}) f_{Q}(\boldsymbol{p}+\boldsymbol{k})-w(\boldsymbol{p}, \boldsymbol{k}) f_{Q}(\boldsymbol{p})\right],
$$

where $w$ is the transition rate of heavy quark colliding with heat bath particles of momentum $\boldsymbol{k}$. The first term in Eq. (3) is the gain term that describes the transition of HF quark from a state of momentum $\boldsymbol{p}+\boldsymbol{k}$ to momentum state $\boldsymbol{p}$ while the loss term (second term) represents the scattering out from the momentum state $\boldsymbol{p}$. Assuming the scatterings of HF quark with the bulk medium partons is dominated by small momentum transfer i.e., $|\boldsymbol{k}| \ll|\boldsymbol{p}|$, the distribution function of HQ and transition rate can be expanded up to second order with respect to $\boldsymbol{k}$ i.e., 


$$
\begin{aligned}
w(\boldsymbol{p}+\boldsymbol{k}, \boldsymbol{k}) f_{Q}(\boldsymbol{p}+\boldsymbol{k}) \\
\simeq w(\boldsymbol{p}, \boldsymbol{k}) f_{Q}(\boldsymbol{p})+\boldsymbol{k} \frac{\partial}{\partial \boldsymbol{p}}\left[w(\boldsymbol{p}, \boldsymbol{k}) f_{Q}(\boldsymbol{p})\right] \\
\quad+\frac{1}{2} k_{i} k_{j} \frac{\partial^{2}}{\partial p_{i} \partial p_{j}}\left[w(\boldsymbol{p}, \boldsymbol{k}) f_{Q}(\boldsymbol{p})\right] .
\end{aligned}
$$

With this approximation the collision integral simplifies to

$C\left[f_{Q}\right]=\int d^{3} k\left[k_{j} \frac{\partial}{\partial p_{j}}+\frac{1}{2} k_{i} k_{j} \frac{\partial^{2}}{\partial p_{i} \partial p_{j}}\right] w(\boldsymbol{p}, \boldsymbol{k}) f_{Q}(\boldsymbol{p})$.

The function $w$ can be expressed in terms of the crosssection for scattering processes in the heat bath. For, scattering of HQ with momentum $p$ with the bulk medium-light thermal parton with momentum $\boldsymbol{q}$, one finds

$$
w(\boldsymbol{p}, \boldsymbol{k})=\gamma_{l} \int \frac{d^{3} q}{(2 \pi)^{3}} f(q)_{l}\left|\boldsymbol{v}_{\mathrm{rel}}\right| \frac{d \sigma}{d \boldsymbol{\Omega}}(\boldsymbol{p}, \boldsymbol{q} \rightarrow \boldsymbol{p}-\boldsymbol{k}, \boldsymbol{q}+\boldsymbol{k}),
$$

where $f(q)_{l}$ is Fermi-Dirac/or Bose-Einstein distribution function of light thermal partons and $\gamma_{l}$ is degeneracy factor which is $\gamma_{q}=6$ for quarks and $\gamma_{g}=16$ for gluons. Boltzmann equation Eq. (2) can be approximated as Fokker-Planck equation

$$
\frac{\partial}{\partial t} f_{Q}(\boldsymbol{p}, t)=\frac{\partial}{\partial p_{i}}\left(A_{i}(\boldsymbol{p}) f_{Q}(\boldsymbol{p}, t)+\frac{\partial}{\partial p_{j}} B_{i j}(\boldsymbol{p}) f_{Q}(\boldsymbol{p}, t)\right) .
$$

Here $A_{i}$ and $B_{i j}$ are the drag and diffusion coefficients and are given as

$$
\begin{gathered}
A_{i}(\boldsymbol{p})=\int d^{3} k w(\boldsymbol{p}, \boldsymbol{k}) k_{i} \\
B_{i j}(\boldsymbol{p})=\frac{1}{2} \int d^{3} k w(\boldsymbol{p}, \boldsymbol{k}) k_{i} k_{j} .
\end{gathered}
$$

For an isotropic heat bath at local thermal equilibrium one may define [43]

$$
\begin{gathered}
A_{i}(\boldsymbol{p})=A(\boldsymbol{p}) p_{i}, \\
B_{i j}(\boldsymbol{p})=B_{0}(\boldsymbol{p}) P_{i j}^{\|}+B_{1}(\boldsymbol{p}) P_{i j}^{\perp},
\end{gathered}
$$

where $P_{i j}^{\|}$and $P_{i j}^{\perp}$ are longitudinal and transverse projection operators defined as

$$
P_{i j}^{\|}=\frac{p_{i} p_{j}}{|\boldsymbol{p}|^{2}}, \quad P_{i j}^{\perp}=\delta_{i j}-\frac{p_{i} p_{j}}{|\boldsymbol{p}|^{2}} .
$$

For a process $l Q \rightarrow l Q$ (where $l$ stands for light quarks and gluon) the drag and diffusion coefficients of HQ in the plasma of light quarks and gluons are given by the scalar integral of form

$$
\begin{aligned}
\left\langle X\left(\boldsymbol{p}^{\prime}\right)\right\rangle= & \frac{1}{2 E_{p}} \int \frac{d^{3} q}{(2 \pi)^{3} 2 E_{q}} \int \frac{d^{3} p^{\prime}}{(2 \pi)^{3} 2 E_{p^{\prime}}} \int \frac{d^{3} q^{\prime}}{(2 \pi)^{3} 2 E_{q^{\prime}}}|\mathcal{M}|^{2} \\
& \times(2 \pi)^{4} \delta^{4}\left(p+q-p^{\prime}-q^{\prime}\right) f_{l}(q)\left(1 \pm f_{l}(q)\right) X\left(\boldsymbol{p}^{\prime}\right),
\end{aligned}
$$

where $l=q, \bar{q}, g$. In the present study, we evaluate scattering amplitude for relevant $2 \rightarrow 2$ processes within the matrix model which make the matrix element squared color dependent. So in the presence of a background gauge field Eq. (13) becomes

$$
\begin{aligned}
\left\langle X\left(\boldsymbol{p}^{\prime}\right)\right\rangle= & \frac{1}{2 E_{p}} \int \frac{d^{3} q}{(2 \pi)^{3} 2 E_{q}} \int \frac{d^{3} p^{\prime}}{(2 \pi)^{3} 2 E_{p^{\prime}}} \int \frac{d^{3} q^{\prime}}{(2 \pi)^{3} 2 E_{q^{\prime}}} \\
& \times\left(\sum_{a, e}\left|\mathcal{M}_{q Q}\right|_{a b}^{2} f_{a}(q)\left(1-f_{e}\left(q^{\prime}\right)\right)\right. \\
& \left.+\sum_{e, f, g, h}\left|\mathcal{M}_{g Q}\right|_{e f g h}^{2} f_{e f}(q)\left(1+f_{g h}\left(q^{\prime}\right)\right)\right) \\
& \times(2 \pi)^{4} \delta^{4}\left(p+q-p^{\prime}-q^{\prime}\right) X\left(\boldsymbol{p}^{\prime}\right),
\end{aligned}
$$

where $a, e$ are color indices of incoming and outgoing light quark and $e f, g h$ are color indices for incoming and outgoing gluon that interact with $\mathrm{HQ},\left|\mathcal{M}_{q Q}\right|_{a b}^{2}$ and $\left|\mathcal{M}_{g Q}\right|_{\text {efgh }}^{2}$ are matrix element squared respectively for the processes $q^{a} Q^{c} \rightarrow q^{b} Q^{d}$ and $g^{e f} Q^{a} \rightarrow g^{g h} Q^{b}$. In the notation as written in Eq. (14), the drag and diffusion coefficients are written as

$$
\begin{gathered}
A(\boldsymbol{p})=\langle 1\rangle-\frac{\left\langle\boldsymbol{p} \cdot \boldsymbol{p}^{\prime}\right\rangle}{|\boldsymbol{p}|^{2}} \\
B_{0}(\boldsymbol{p})=\frac{1}{4}\left(\left\langle\left.\boldsymbol{p}^{\prime}\right|^{2}\right\rangle-\frac{\left\langle\left(\boldsymbol{p} \cdot \boldsymbol{p}^{\prime}\right)^{2}\right\rangle}{|\boldsymbol{p}|^{2}}\right) \\
B_{1}(\boldsymbol{p})=\frac{1}{2}\left(\frac{\left\langle\left(\boldsymbol{p} \cdot \boldsymbol{p}^{\prime}\right)^{2}\right\rangle}{|\boldsymbol{p}|^{2}}-2\left\langle\boldsymbol{p} \cdot \boldsymbol{p}^{\prime}\right\rangle+|\boldsymbol{p}|^{2}\langle 1\rangle\right) .
\end{gathered}
$$

In the presence of a nontrivial Polyakov loop background, apart from the matrix elements, the distribution functions also become color dependent. We evaluate these scattering amplitudes in the matrix model of semi-QGP which we discuss in the next section.

\section{SEMI-QGP}

At high temperature, the density of colored particles like quarks and gluon are large and can be calculated using perturbative QCD. However, at low temperature, colored 
particles are statistically suppressed and are measured by the small value of the Polyakov loop, e.g., at chiral crossover temperature $T_{c} \sim 170 \mathrm{MeV}, \phi=0.2$ [44] which is way smaller from its asymptotic value i.e., $\phi=1$. Because of the suppression of colored particles, the region near chiral cross-over is termed as semi-QGP [45]. Semi-QGP is characterized by the Polyakov loop as defined in Eq. (24). For the calculational purpose, we shall use double line notation which is quite useful in the matrix model of semi QGP. In the double line basis, quark carries one color index say $a=1,2, \ldots, N$ and gluons carry double index say $a b=1,2, . ., N^{2}$. For $S U(N)$ group such $N^{2}$ pairs lead to $N^{2}$ generators and the basis is overcomplete by one generator. The overcomplete basis is compensated by introducing the projection operator defined as [46-48]

$$
\mathcal{P}_{c d}^{a b}=\mathcal{P}_{b a ; c d}=\mathcal{P}^{a b ; d c}=\delta_{c}^{a} \delta_{d}^{b}-\frac{1}{N} \delta^{a b} \delta_{c d}
$$

hence the generator is given by

$$
\left(t^{a b}\right)_{c d}=\frac{1}{\sqrt{2}} \mathcal{P}_{c d}^{a b}
$$

The trace over two generators does not vanish but rather is again a projection operator, i.e.,

$$
\operatorname{Tr}\left(t^{a b} t^{c d}\right)=\frac{1}{2} \mathcal{P}^{a b c d} .
$$

This is due to the presence of extra generator as compared to generators in an orthonormal basis. The structure constant of the group in the double line basis is given by

$$
f^{a b, c d, e f}=\frac{i}{\sqrt{2}}\left(\delta^{a d} \delta^{c f} \delta^{e b}-\delta^{a f} \delta^{c b} \delta^{e d}\right) .
$$

In the mean-field approximation, the constant background field is defined as $A_{\mu}^{0}=\frac{1}{g} \delta_{\mu 0} Q^{a}$ with $Q^{a}=2 \pi q^{a} T$. Since $A_{0}$ is traceless so sum over $Q^{\prime}$ 's vanishes i.e., $\sum_{a} Q^{a}=0$. For an $S U(3)$ group, $Q^{a}=\left(-Q^{i},-Q^{i-1}, \ldots 0, Q^{i-1}, Q^{i}\right)$, where $i=N / 2$ if $N$ is even and $(N-1) / 2$ if $N$ is odd. In the temporal direction, the Wilson line is written as

$$
P=\mathcal{P} \exp \left(i g \int_{0}^{\beta} d \tau A_{0}\left(x_{0}, \boldsymbol{x}\right)\right)
$$

where $\mathcal{P}$ stands for the ordering of imaginary time and $\tau$ is imaginary time. Polyakov loop, which is the trace of Wilson line, in the constant background gauge field can be written as

$$
\phi=\frac{1}{N} \sum_{a=1}^{N} \exp \left(i 2 \pi q^{a}\right) .
$$

For an $S U(3)$ group, where $q^{a}=(-q, 0, q)$ Eq. (23) is simplified to

$$
\phi=\frac{1}{3}(1+2 \cos (2 \pi q)) .
$$

The background gauge field acts as an imaginary chemical potential for colored particles so the statistical distribution function of quark/antiquark and the gluon are

$$
\begin{gathered}
f_{a}^{0}(E)=\frac{1}{e^{\beta\left(E-i Q_{a}\right)}+1}, \quad \tilde{f}_{a}^{0}(E)=\frac{1}{e^{\beta\left(E+i Q_{a}\right)}+1}, \\
f_{a b}^{0}(E)=\frac{1}{e^{\beta\left(E-i\left(Q_{a}-Q_{b}\right)\right)}-1},
\end{gathered}
$$

where the single and double indices are for quark/antiquark and gluon. For a background field and given $Q^{a}$ these distribution functions are complex so are unphysical. Physical meaning comes when one integrates over all distributions of $Q^{a}$. The resummed gluon propagator in the presence of a static background gauge field is given as [49]

$D \mu \nu ; a b c d(K)=P_{\mu \nu}^{L} \frac{k^{2}}{K^{2}} D_{a b c d}^{L}(K)+P_{\mu \nu}^{T} D_{a b c d}^{T}(K)$,

where $P_{\mu \nu}^{T}=g_{\mu i}\left(-g^{i j}-\frac{k^{i} k^{j}}{K^{2}}\right) g_{j \nu}$ and $P_{\mu \nu}^{L}=-g_{\mu \nu}+\frac{k_{\mu} k_{\nu}}{K^{2}}-$ $P_{\mu \nu}^{T}$ are the longitudinal and the transverse projection operators. The longitudinal and the transverse gluon propagators are written as

$$
\begin{aligned}
& D_{\mu \nu ; a b c d}^{L}(K)=\left(\frac{i}{K^{2}+F}\right)_{a b c d}, \\
& D_{\mu \nu ; a b c d}^{T}(K)=\left(\frac{i}{K^{2}-G}\right)_{a b c d},
\end{aligned}
$$

where

$$
\begin{gathered}
F=2 M^{2}\left(1-\frac{y}{2} \ln \left(\frac{y+1}{y-1}\right)\right), \\
G=M^{2}\left(y^{2}+\frac{y\left(1-y^{2}\right)}{2} \ln \left(\frac{y+1}{y-1}\right)\right),
\end{gathered}
$$

with $y=\frac{k_{0}}{|k|}$ and $M^{2}=\left(M^{2}\right)_{a b c d}$ is the thermal mass of the gluon. For the drag and the diffusion of HQ studied here, the momentum transfer is small so only longitudinal propagator contributes to the squared matrix elements $[43,50]$. 


\section{VISCOUS CORRECTIONS IN THE DISTRIBUTION FUNCTIONS}

In this section, we briefly describe the first order viscous corrections on the thermal distribution function of quarks and gluons. We start with the energy-momentum tensor of a nonideal fluid which is given as [31]

$$
T^{\mu \nu}=(\epsilon+P) u^{\mu} u^{\nu}+P g^{\mu \nu}+\pi^{\mu \nu}+\Pi \nabla^{\mu \nu},
$$

where $\epsilon, P, u^{\mu}$ are the energy density, pressure density and four-velocity of the fluid. For metric tensor, we use the convention $g^{\mu \nu}=\operatorname{diag}(-1,+1,+1,+1)$ so that $u^{\mu} u_{\mu}=-1$ and the term $\nabla^{\mu \nu}=g^{\mu \nu}+u^{\mu} u^{\nu}$. The first two terms at the right hand side of Eq. (32) describes the energy-momentum tensor for an ideal fluid and the rest two terms are part of viscous corrections that summarizes the effect of shear and bulk viscosities respectively. The dissipative terms are constructed from the derivatives $\Delta^{\alpha}=\nabla^{\alpha \beta} \partial_{\beta}$ and $\nabla^{\mu \nu}$. In the first-order approximation, the symmetric tensor $\pi^{\mu \nu}$ satisfying the condition $u_{\mu} \pi^{\mu \nu}=0$, in the local rest frame is given as

$$
\pi^{\mu \nu}=-\eta\left(\Delta^{\mu} u^{\nu}+\Delta^{\nu} u^{\mu}-\frac{2}{3} \nabla^{\mu \nu} \Delta_{\alpha} u^{\alpha}\right)
$$

and the bulk viscosity dependent term

$$
\Pi=-\xi \Delta_{\alpha} u^{\alpha} .
$$

Dissipative effects can be incorporated in the color dependent distribution functions $f_{a / a b}(E)$ which contains the ideal part as well as viscous corrections. For this purpose, we write $f_{a / a b}(E)=f_{a / a b}^{0}(E)+\delta f_{a / a b}(E)\left(f_{a / a b}^{0}(E)\right.$ is equilibrium distribution function of quark/antiquark and gluon) and use the second-moment ansatz as in Refs. $[31,51,52]$, so that

$$
\begin{aligned}
\delta f(E)_{a / a b}= & \frac{1}{T^{3} s} f(E)_{a / a b}^{0}\left(1+f(E)_{a / a b}^{0}\right) p^{\mu} p^{\nu} \\
& \times\left(\frac{A}{2} \pi_{\mu \nu}+\frac{B}{5} \Pi \nabla_{\mu \nu}\right)
\end{aligned}
$$

where $A$ and $B$ are constants. Constrain on $\delta f_{a / a b}$ comes from the continuity of stress-energy tensor across the freeze-out hypersurface [52] i.e.,

$$
\delta T^{\mu \nu}=\int \frac{d^{3} k}{(2 \pi)^{3}} \frac{k^{\mu} k^{\nu}}{E_{k}} \delta f_{a / a b}(E) .
$$

The choice of $\delta f_{a / a b}$ is not unique, as pointed out in Ref. [53], $\delta f_{a / a b}$ can have linearly increasing form with momentum and also quadratically increasing with momentum or anything in between linear to quadratic increasing behavior. However, we will continue with the form as in
Refs. [31,52]. In the local rest frame of the fluid, i.e., $u_{0}=1, u_{i}=0, \partial_{\mu} u_{0}=0$ and $\partial_{\mu} u_{i} \neq 0$, the deviation in distribution function can be written as [31,52]

$$
\begin{aligned}
\delta f(E)_{a / a b}= & \frac{1}{T^{3} s} f(E)_{a / a b}^{0}\left(1 \mp f(E)_{a / a b}^{0}\right) p^{\mu} p^{\nu} \\
& \times\left(\frac{1}{2} \pi_{\mu \nu}+\frac{1}{5} \Pi \nabla_{\mu \nu}\right) .
\end{aligned}
$$

The first term of Eq. (37), i.e., the shear viscosity dependent term is

$p^{\mu} p^{\nu} \pi_{\mu \nu}=-p^{\mu} p^{\nu} \eta\left(\Delta_{\mu} u_{\nu}+\Delta_{\nu} u_{\mu}-\frac{2}{3} \nabla_{\mu \nu}\left(\partial^{\alpha}+u^{\alpha} u^{\beta} \partial_{\beta}\right) u_{\alpha}\right)$.

One can use the normalization condition $u_{\mu} u^{\mu}=-1$ and differentiating this relation leads to $u^{\mu} \partial_{\nu} u_{\nu}=0$. Using this and the relation $\Delta_{\mu}=\nabla_{\mu \nu} u^{\nu}$ one obtains

$$
\begin{aligned}
p^{\mu} p^{\nu} \pi_{\mu \nu}= & -\eta\left(p^{\mu} p^{\nu}\left(\partial_{\mu} u_{\nu}+\partial_{\nu} u_{\mu}\right)+2(p \cdot u) p^{\nu} u^{\beta} \partial_{\beta} u_{\nu}\right. \\
& \left.-\frac{2}{3}\left(p^{2}-(p \cdot u)^{2}\right) \partial^{\alpha} u_{\alpha}\right) .
\end{aligned}
$$

Now, one can further use the relations $\partial_{\mu} u_{\nu}=\left(\tilde{g}_{\mu \nu}-u_{\mu} u_{\nu}\right) /$ $\tau$ and $\partial^{\alpha} u_{\alpha}=1 / \tau$ [54], where $\tau$ is the proper time so that in the local rest frame of the fluid, Eq. (39) reduces to [36]

$$
p^{\mu} p^{\nu} \pi_{\mu \nu}=\frac{2 \eta}{\tau}\left(-p_{z}^{2}+\frac{\mathbf{p}^{2}}{3}\right)
$$

Similarly, the bulk viscosity dependent term can be written as

$$
p^{\mu} p^{\nu} \nabla_{\mu \nu} \Pi=-\xi p^{\mu} p^{\nu}\left(g_{\mu \nu}+u_{\mu} u_{\nu}\right) \Delta_{\alpha} u^{\alpha} .
$$

Using the same relations as for the case of shear viscosity, Eq. (41) can be written as

$$
p^{\mu} p^{\nu} \nabla_{\mu \nu} \Pi=\frac{\xi}{\tau} \mathbf{p}^{2} .
$$

Thus the distribution functions of quarks and gluons of Eq. (37) with the effect of shear and bulk viscosities can be written as

$$
\begin{aligned}
f(E)_{a}=f(E)_{a}^{0}+ & \frac{f(E)_{a}^{0}\left(1-f(E)_{a}^{0}\right)}{T^{3} \tau}\left[\frac{\eta}{s}\left(-p_{z}^{2}+\frac{\mathbf{p}^{2}}{3}\right)+\frac{\xi}{s} \frac{\mathbf{p}^{2}}{5}\right] \\
f(E)_{a b}= & f(E)_{a b}^{0}+\frac{f(E)_{a b}^{0}\left(1+f(E)_{a b}^{0}\right)}{T^{3} \tau} \\
& \times\left[\frac{\eta}{s}\left(-p_{z}^{2}+\frac{\mathbf{p}^{2}}{3}\right)+\frac{\xi}{s} \frac{\mathbf{p}^{2}}{5}\right]
\end{aligned}
$$


In the present investigation, for evaluating drag and diffusion coefficients, we shall use Eqs. (43) and (44) for quark/ antiquark and gluon distribution function in Eq. (14).

\section{SCATTERING AMPLITUDES WITHIN MATRIX MODEL}

In this section, We shall discuss the scattering of HQ of mass $M$ and energy $E=\sqrt{p^{2}+M^{2}}$ with the light thermal partons in the bulk medium and we shall also compute the scattering amplitude squared within the matrix model of semi QGP. To compute the drag and the diffusion coefficients of HQ transport we shall follow a similar approach to include screening effects as in Refs. [42,50]. For the elastic collision, there are two types of scattering processes that contributes to the drag and the diffusion coefficient of HQ. One is Coulomb scattering, i.e., scattering off of HQ with light quark and another is Compton scattering, i.e., scattering off of HQ with gluons. In the following we present these in detail.

\section{A. Coulomb scattering}

The Feynman diagram for the Coulomb scattering of HQ and a light quark is shown on the left side of Fig. 1. Here $a$, $c, b, d$ are the color indices of initial and final quarks. In the double line notation, the scattering amplitude for this process is
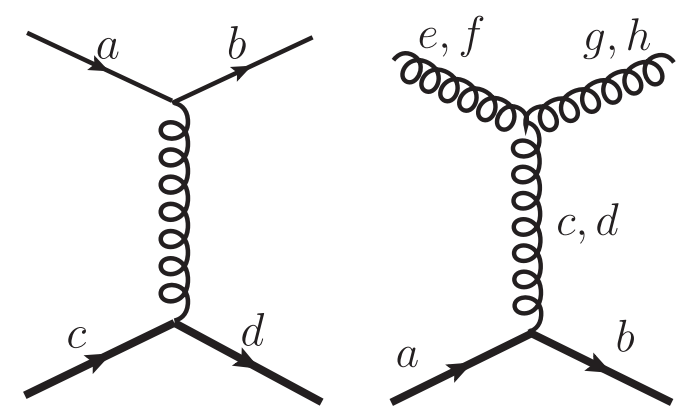

FIG. 1. Coulomb scattering (left) of HQ (bold solid line) and light quark/antiquark (thin solid line). t-channel Compton scattering (right). The curly line represent a gluon.

$$
\begin{aligned}
i \mathcal{M}_{q Q}= & \frac{(i g)^{2}}{\left(t+\left(m_{D}^{2}\right)_{m l j k}\right)}\left(t^{j k}\right)_{a b}\left(t^{m l}\right)_{c d}\left[\bar{u}_{b}\left(q^{\prime}\right) \gamma^{\mu} u_{a}(q)\right] \\
& \times\left[\bar{u}_{d}\left(p^{\prime}\right) \gamma_{\mu} u_{c}(p)\right],
\end{aligned}
$$

where $g$ is coupling constant, $t$ is Mandelstam variable and $m, l, j, k$ are the color indices of gluon propagator. In the limit of soft momentum transfer, only timelike component of the propagator contributes and the propagators simply become Debye screened propagator with $1 / t \rightarrow 1 /\left(t+m_{D}^{2}\right)$ $[42,50]$ where $m_{D}^{2}$ is color dependent Debye mass and can be given as

$$
\begin{aligned}
\left(m_{D}^{2}\right)_{a b c d}= & \frac{g^{2}}{6}\left[\delta_{a d} \delta_{b c}\left(\sum_{e=1}^{3}\left(\mathcal{D}\left(Q_{a e}\right)+\mathcal{D}\left(Q_{e b}\right)\right)-N_{f}\left(\tilde{\mathcal{D}}\left(Q_{a}\right)+\tilde{\mathcal{D}}\left(Q_{b}\right)\right)\right)\right. \\
& \left.-2 \delta_{a b} \delta_{c d}\left(\mathcal{D}\left(Q_{a c}\right)-\frac{N_{f}}{N}\left(\tilde{\mathcal{D}}\left(Q_{a}\right)+\tilde{\mathcal{D}}\left(Q_{c}\right)\right)+\frac{N_{f}}{N^{2}} \sum_{e=1}^{3} \tilde{\mathcal{D}}\left(Q_{e}\right)\right)\right],
\end{aligned}
$$

where

$$
\mathcal{D}\left(Q_{a}\right)=\frac{3}{\pi^{2}} \int_{0}^{\infty} d E E\left(\frac{1}{e^{\beta\left(E+i Q_{a}\right)}-1}+\frac{1}{e^{\beta\left(E-i Q_{a}\right)}-1}\right),
$$

and $\tilde{\mathcal{D}}\left(Q_{a}\right)=\mathcal{D}\left(Q_{a}+\pi T\right)$. In the perturbative limit, Eq. (45) can be written as

$$
i \mathcal{M}_{q Q}=-\frac{g^{2}}{t}\left(t^{j k}\right)_{a b}\left(t^{j k}\right)_{c d}\left[\bar{u}_{b}\left(q^{\prime}\right) \gamma^{\mu} u_{a}(q)\right]\left[\bar{u}_{d}\left(p^{\prime}\right) \gamma_{\mu} u_{c}(p)\right],
$$

and the product of projection operator with open color index $a, b$ can be written as

$$
\mathcal{P}_{a b}^{j k} \mathcal{P}_{c d}^{j k} \mathcal{P}_{b a}^{j^{\prime} k^{\prime}} \mathcal{P}_{d c}^{j^{\prime} k^{\prime}}=(N-1)\left(1-\frac{\delta_{b a}}{N}\right)
$$

However, for the computation of the drag and the diffusion coefficient, we shall use Eq. (45). Simplifying Eq. (45) for massless light quark and massive heavy quark by summing and averaging over final and initial spins, the scattering amplitude squared $\left(\left|\mathcal{M}_{q Q}\right|^{2}\right)$ can be written as

$$
\left|\mathcal{M}_{q Q}\right|^{2}=\frac{g^{4}}{16 N_{c}^{2}} \mathcal{P}_{a b}^{j k} \mathcal{P}_{c d}^{m l} \mathcal{P}_{b a}^{j^{\prime} k^{\prime}} \mathcal{P}_{d c}^{m^{\prime} l^{\prime}} \frac{\left(8\left(s-M^{2}\right)^{2}+8\left(u-M^{2}\right)^{2}+16 M^{2} t\right)}{\left(t+\left(m_{D}^{2}\right)_{m l j k}\right)\left(t+\left(m_{D}^{2}\right)_{m^{\prime} l^{\prime} j^{\prime} k^{\prime}}\right)}
$$


Let us note here that the drag and the diffusion coefficient of HQ as defined in Eqs. (15) and (16) depends on the color of incoming and outgoing light quark, i.e., $Q^{a}$ and $Q^{b}$ in the distribution functions; see Eq. (14). So to compute the coloraveraged quantity, the color index $a$ and $b$ in Eq. (50) will be summed with the distribution function. With the distribution function as defined in Eq. (43), for Coulomb scattering the bracketed quantity in Eq. (14) becomes

$$
\left\langle X\left(p^{\prime}\right)\right\rangle=\frac{1}{2 E_{p}} \sum_{a b c d} \int \frac{d^{3} q}{2 E_{q}(2 \pi)^{3}} \frac{d^{3} p^{\prime}}{2 E_{p^{\prime}}(2 \pi)^{3}} \frac{d^{3} q^{\prime}}{2 E_{q^{\prime}}(2 \pi)^{3}} \sum_{j k j^{\prime} k^{\prime}} \sum_{m l m^{\prime} l^{\prime}}\left|\mathcal{M}_{q Q}\right|^{2}\left(f_{a}^{0}(q)+\delta f_{a}(q)\right)\left(1-f_{b}^{0}\left(q^{\prime}\right)-\delta f_{b}\left(q^{\prime}\right)\right)\left\langle X\left(p^{\prime}\right)\right\rangle
$$

\section{B. Compton scattering}

There are three types of scatterings ( $s, t$, and $u$ channels) that contribute to the Compton scattering, i.e., scattering off of a gluon from a quark. For $s$ and $u$ channel scatterings, the corresponding Feynman diagrams are shown in Fig. 2 and for the $t$ channel scattering the relevant diagram is shown in right side of Fig. 1. We shall evaluate the scattering amplitude for Compton scattering below.

\section{C. s-channel}

The relevant diagram for this channel is shown on the left side of Fig. 2 where $e f(g h), a(b)$ are color indices of incoming (outgoing) gluon and quark. In the double line notation, the scattering amplitude for the process is given as

$i \mathcal{M}_{s}=i(i g)^{2}\left(t^{e f}\right)_{a c}\left(t^{g h}\right)_{c b}\left[\frac{\bar{u}_{b}\left(p^{\prime}\right) \phi(\not p+\not 1+M) \phi u_{a}(p)}{s-M^{2}}\right]$.

where $s$ is Mandelstam variable and $M$ is the mass of HQ. Note here that unlike Coulomb scattering there is no color dependence on the HQ propagator. This is because of the large mass of heavy quark. For massive quark and massless gluon the matrix element squared for s-channel Compton scattering can be written as

$$
\begin{aligned}
\left|\mathcal{M}_{s}\right|^{2}= & \frac{8 g^{4}}{16 N_{c}\left(N_{c}^{2}-1\right)} \mathcal{P}_{a c}^{e f} \mathcal{P}_{a c^{\prime}}^{e f} \mathcal{P}_{c b}^{g h} \mathcal{P}_{c^{\prime} b}^{g h} \\
& \times\left(\frac{M^{2}\left(M^{2}-u-3 s\right)-u s}{\left(s-M^{2}\right)^{2}}\right) .
\end{aligned}
$$

Note that the scattering amplitude depends on the color of quarks and gluons. For the evaluation of the transport coefficients one needs to perform a color sum. Same as in
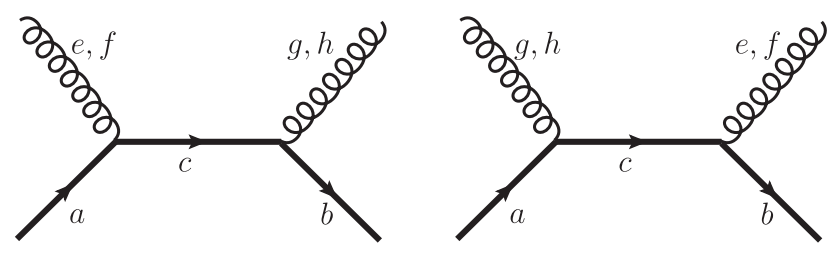

FIG. 2. s-channel Compton scattering (left). u-channel Compton scattering (right). the case of Coulomb scattering, the color indices of incoming and outgoing gluon $(e f, g h)$ in Eq. (53) will be summed with the distribution functions appearing in Eq. (14).

\section{D. u channel}

The corresponding Feynman diagram for $u$ channel Compton scattering is illustrated at the right side of Fig. 2. Scattering amplitude that depends on the color of incoming and outgoing color particles can be written as

$$
i \mathcal{M}_{u}=i(i g)^{2}\left(t^{e f}\right)_{c b}\left(t^{g h}\right)_{a c}\left[\frac{\bar{u}_{b}\left(p^{\prime}\right) \phi\left(\not p-\not{ }^{\prime}+M\right) \phi u_{a}(p)}{u-M^{2}}\right] .
$$

Simplifying Eq. (54) with the polarization sum of massless gluon and spin sum and average over heavy quark gives

$$
\begin{aligned}
\left|\mathcal{M}_{u}\right|^{2}= & \frac{8 g^{4}}{16 N_{c}\left(N_{c}^{2}-1\right)} \mathcal{P}_{a c}^{g h} \mathcal{P}_{a c^{\prime}}^{g h} \mathcal{P}_{c b}^{e f} \mathcal{P}_{c^{\prime} b}^{e f} \\
& \times\left(\frac{M^{2}\left(M^{2}-3 u-s\right)-u s}{\left(u-M^{2}\right)^{2}}\right) .
\end{aligned}
$$

In the Eq. (55), the product of projection operator can be simplified by summing over the color indices $a, b$ and $c$. However, the color indices of initial and final gluon should be summed with the distribution function in Eq. (14). Keeping ef, $g h$ as open indices, the product of the projection operators can be simplified to

$$
\begin{aligned}
\mathcal{P}_{a c}^{g h} \mathcal{P}_{a c^{\prime}}^{g h} \mathcal{P}_{c b}^{e f} \mathcal{P}_{c^{\prime} b}^{e f}= & \delta_{e h}-\frac{1}{N_{c}}\left(2 \delta_{e f} \delta_{f h} \delta_{e h}+\delta_{g h} \delta_{e g} \delta_{e h}\right) \\
& +\frac{1}{N_{c}^{2}}\left(\delta_{e f}+\delta_{e f} \delta_{g f} \delta_{e h} \delta_{g h}+\delta_{e f} \delta_{f h} \delta_{e g} \delta_{g h}\right. \\
& \left.+\delta_{e h} \delta_{f g} \delta_{e f} \delta_{g h}+\delta_{g h}\right) \\
& -\frac{1}{N_{c}^{3}}\left(\delta_{e f} \delta_{g h}+\delta_{e f} \delta_{e h} \delta_{g h}\right)
\end{aligned}
$$

\section{E. $\mathbf{t}$ channel}

The relevant Feynman diagram for the $t$ channel Compton scattering is shown on the right side of Fig. 1. For the color dependent scattering amplitude one can write 


$$
\begin{aligned}
i \mathcal{M}_{t}= & (i g)^{2}\left(t^{m l}\right)_{a b} f^{c d, e f, g h} \\
& \times\left[\frac{\epsilon_{\mu}(q) \epsilon_{\nu}^{*}\left(q^{\prime}\right) C^{\mu \alpha \nu}\left(q-q^{\prime},-q,-q^{\prime}\right) \bar{u}_{b}\left(p^{\prime}\right) \gamma_{\alpha} u_{a}(p)}{\left(t+\left(m_{D}^{2}\right)_{m l c d}\right)}\right],
\end{aligned}
$$

where

$$
\begin{aligned}
& C^{\mu \nu \rho}\left(k_{1}, k_{2}, k_{3}\right) \\
& =\left[\left(k_{1}-k_{2}\right)^{\rho} g^{\mu \nu}+\left(k_{2}-k_{3}\right)^{\mu} g^{\nu \rho}+\left(k_{3}-k_{1}\right)^{\nu} g^{\mu \rho}\right] .
\end{aligned}
$$

In Eq. (57), $f^{c d, e f, g h}$ is structure constant as defined in Eq. (21) and $\epsilon_{\mu}(q), \epsilon_{\nu}^{*}\left(q^{\prime}\right)$ are the polarization vectors for incoming and outgoing gluon. The matrix element squared can be obtained by performing appropriate polarization sum for gluons and spin sum and average for heavy quark. Doing so, matrix element squared becomes

$$
\begin{aligned}
\left|\mathcal{M}_{t}\right|^{2}= & \frac{16 g^{4}}{8 N\left(N^{2}-1\right)} \mathcal{P}_{a b}^{m l} \mathcal{P}_{b a}^{l^{\prime} m^{\prime}} f^{c d, e f, g h} f^{d^{\prime} c^{\prime}, f e, h g} \\
& \times\left(\frac{-\left(M^{2}-s\right)\left(M^{2}-u\right)}{\left(t+\left(m_{D}^{2}\right)_{m l c d}\right)\left(t+\left(m_{D}^{2}\right)_{m^{\prime} l^{\prime} c^{\prime} d^{\prime}}\right)}\right) .
\end{aligned}
$$

The corresponding interference terms among Compton scatterings are given in the Appendix A. To that the total scattering amplitude of Compton scattering that enters in Eq. (14) for evaluation of the drag and the diffusion coefficients is $\left|\mathcal{M}_{g Q}\right|_{\text {efgh }}^{2}=\left|\mathcal{M}_{s}\right|^{2}+\left|\mathcal{M}_{u}\right|^{2}+\left|\mathcal{M}_{t}\right|^{2}+$ $\left|\mathcal{M}_{s}\right|^{\dagger}\left|\mathcal{M}_{u}\right|+\left|\mathcal{M}_{u}\right|^{\dagger}|\mathcal{M}|_{s}+\left|\mathcal{M}_{s}\right|^{\dagger}\left|\mathcal{M}_{t}\right|+\left|\mathcal{M}_{t}\right|^{\dagger}|\mathcal{M}|_{s} \mid+$ $\left|\mathcal{M}_{t}\right|^{\dagger}\left|\mathcal{M}_{u}\right|+\left|\mathcal{M}_{u}\right|^{\dagger}|\mathcal{M}|_{t}$. For computational simplification, we shall use the leading order contribution in the Debye mass that appears in the $t$ channel scatterings.

\section{RESULTS AND DISCUSSIONS}

With the scattering amplitude for the processes $l Q \rightarrow l Q$ (where $l$ stands for light quark/antiquark and gluon and $Q$ stands for HQ) as evaluated in the previous section, we numerically compute the drag and the diffusion coefficients using Eq. (14) and incorporate the dissipative effects in the quark/antiquark and gluon color distribution functions as defined in Eqs. (43) and (44). For this purpose, we use charm quark mass $M=1.27 \mathrm{GeV}$ and the two loop running coupling constant [55]

$$
\alpha_{s}=\frac{1}{4 \pi} \frac{1}{2 \beta_{0} \ln \frac{\pi T}{\Lambda}+\frac{\beta_{1}}{\beta_{0}} \ln \left(2 \ln \left(\frac{\pi T}{\Lambda}\right)\right)}
$$

where

$$
\begin{gathered}
\beta_{0}=\frac{1}{16 \pi^{2}}\left(11-\frac{2 N_{f}}{3}\right) \\
\beta_{1}=\frac{1}{\left(16 \pi^{2}\right)^{2}}\left(102-\frac{38 N_{f}}{3}\right)
\end{gathered}
$$

with $\Lambda=260 \mathrm{MeV}$ and light quark flavor $N_{f}=2$. We also evaluate the HQ transport coefficients in $\mathrm{pQCD}$ by evaluating scattering amplitude squared within the $\mathrm{pQCD}$ framework.

In general, there are two factors that essentially affect the heavy quark transport properties. One is the Debye mass that appears in the evaluation of the matrix elements and the other is the Polyakov loop dependent distribution functions of quark/anti-quark and gluon. At low temperature, a lower value of the Debye mass increases the transport coefficients. On the other hand, the distribution function with the nontrivial $\phi$ tend to reduce it. Apart from these, a third factor that plays an important role here is the momentum dependence of departure $\delta f_{a / a b}$ in Eqs. (43) and (44) of the distribution function from the equilibrium distribution function. Now let us examine the results in some detail. In Fig. 3, we show the dependence of the drag coefficient as a function of temperature. In the left panel, we have plotted the drag coefficient [Eq. (15)] for a constant value of $\eta / s$ and $\xi / s=0$ normalized to the drag coefficient for $\eta / s=0$, $\xi / s=0$ i.e., $A(\eta) / A(\eta=0)$. In both the figures of Fig. 3, we have taken $\tau=0.3 \mathrm{fm}^{-1}$ and the HQ momentum $p=1 \mathrm{GeV}$. The blue curve corresponds to the pQCD results and the red curve corresponds to the effect of the Polyakov loop within the matrix model. It is clear that at low temperature, for $\eta / s=0.1$ and $\tau=0.3 \mathrm{fm}^{-1}$, the drag coefficient is small within the matrix model compared to $\mathrm{pQCD}$. As the temperature increases the suppression in the drag coefficient decreases and approaches the perturbative value at high temperature beyond which it decreases similar to the perturbative results. This nonmonotonic behavior is mainly because of the negative contribution from the momentum factor $\left(\mathbf{q}^{2} / 3-q_{z}^{2}\right)$ in $\delta f_{a / a b}$ and can be understood as follows. In the Polyakov loop background, a smaller value of the Debye mass at low temperature lead to more negative contribution due to the momentum factor in $\delta f_{a / a b}$ and hence smaller drag coefficient with finite $\eta / s$. Another reason for more suppression in the drag coefficient within the matrix model is due to the distribution function i.e., colored particles are suppressed due to small value of Polyakov loop compared to pQCD. In the right panel of Fig. 3, the temperature behavior of the normalized drag coefficient $(A(\xi) / A(\xi=0))$ is shown for $\xi / s=0.03$ and $\eta / s=0$. It can be observed that with the inclusion of the bulk viscosity the drag coefficient is large compared to the $\xi / s=0$ case. The drag coefficient within the matrix model is large compared to $\mathrm{pQCD}$. This is because the $\xi / s$ term in $\delta f_{a / a b}$ is always positive so the smaller value of the Debye mass within the matrix model enhances the drag coefficient.

The ratio $A(\eta) / A(\eta=0)$ of the drag coefficient as defined in Eq. (15) is plotted as a function of temperature for HQ momentum $p=1 \mathrm{GeV}$ in Fig. 4 for various value of $\eta / s$ and $\tau$ to see the effect of both $(\eta / s, \tau)$ the quantities. Here the scattering amplitude squared for the relevant scatterings are evaluated within the matrix model. As 

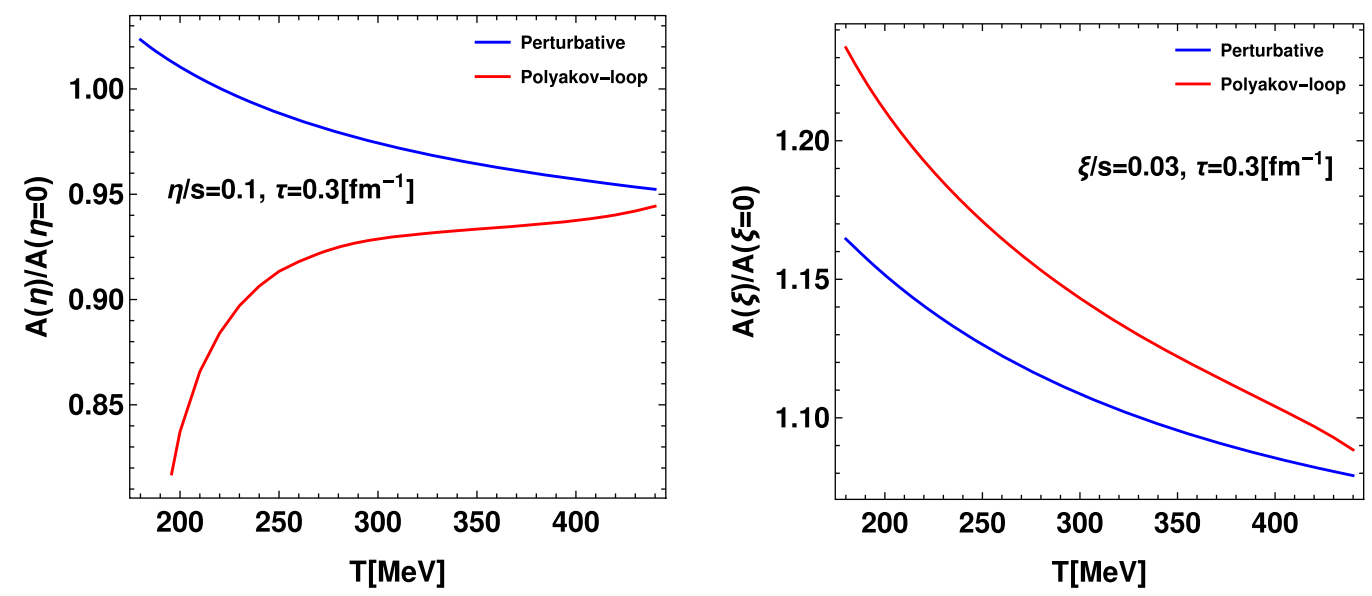

FIG. 3. Left panel: The ratio of the drag coefficient i.e., $A(\eta) / A(\eta=0)$ is shown as a function of temperature for $p=1 \mathrm{GeV}$, $\eta / s=0.1, \tau=0.3 \mathrm{fm}^{-1}$ and $\xi / s=0$. The blue curve represents the perturbative QCD result while the red curve corresponds to including Polyakov loop within the matrix model. Right panel: The ratio $A(\xi) / A(\xi=0)$ is shown as a function of temperature for $\xi / s=0.03, p=1 \mathrm{GeV}, \tau=0.3 \mathrm{fm}^{-1}$ and $\eta / s=0$. Similar to the left panel, the blue curve corresponds to pQCD results and the red curve corresponds to including Polyakov loop within the matrix model.
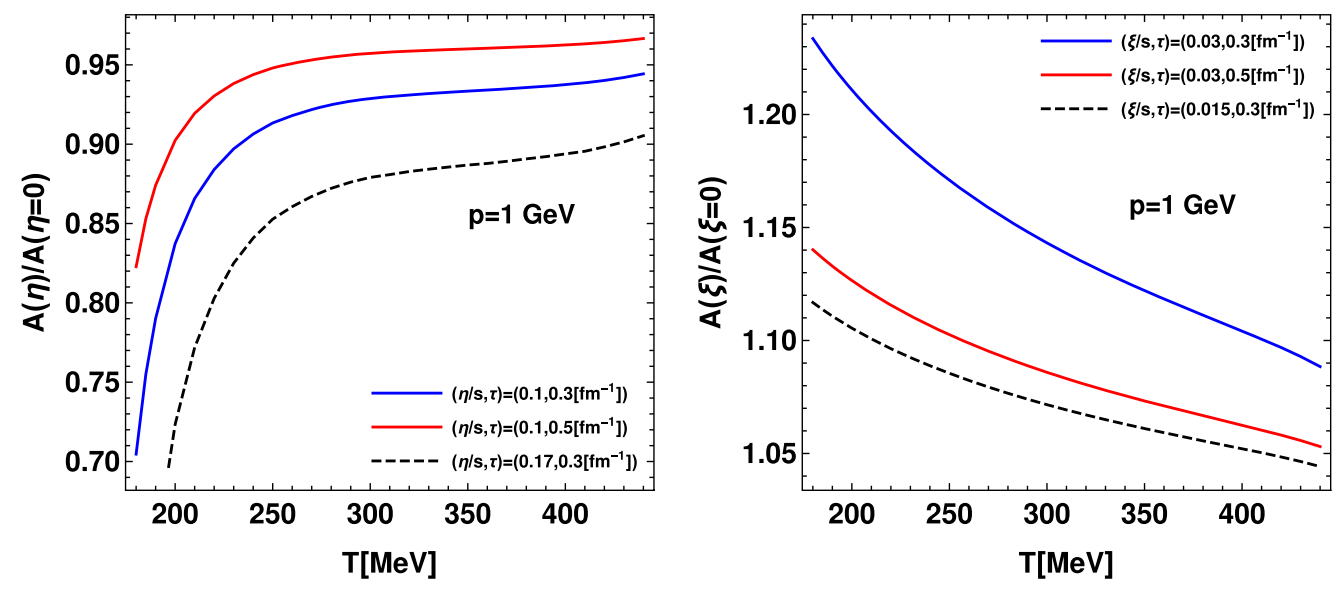

FIG. 4. Left panel: The ratio $A(\eta) / A(\eta=0)$ as a function of temperature for HQ momentum $p=1 \mathrm{GeV}$ and $\xi / s=0$. The topmost curve is for $\eta / s=0.1, \tau=0.3 \mathrm{fm}^{-1}$, bottom-most, i.e., dashed black curve is for $\eta / s=0.17, \tau=0.3 \mathrm{fm}^{-1}$ and the blue curve is for $\eta / s=0.1, \tau=0.5 \mathrm{fm}^{-1}$. Right panel: The ratio of the drag coefficient $A(\xi) / A(\xi=0)$ as a function of temperature for HQ momentum $p=1 \mathrm{GeV}$ and $\eta / s=0$. The blue curve corresponds to $\xi / s=0.03, \tau=0.3 \mathrm{fm}^{-1}$, the red curve corresponds to $\xi / s=0.03, \tau=$ $0.5 \mathrm{fm}^{-1}$ and the dashed black curve corresponds to $\xi / s=0.015, \tau=0.3 \mathrm{fm}^{-1}$.

anticipated from the effect of phase space (momentum dependent term in $\delta f_{a / a b}$ ), Polyakov loop dependent distribution functions of quark/antiquark and gluon, and the Debye mass, with an increase in $\eta / s$ the HQ drag coefficient decreases as shown by the black dashed and the blue curves on the left panel of Fig. 4. Here the blue line is for $\eta / s=0.1$ and black dashed line for $\eta / s=0.17$ with $\tau=0.3 \mathrm{fm}^{-1}$. With increase in the proper time the drag coefficient increases which is shown by the red and the blue curve of the same figure. This can be understood from $1 / \tau$ factor in Eqs. (25) and (26). It is also observed that, for small value of $\eta / s$ and sufficiently large value of $\tau$, the effect of $\eta / s$ on the HQ drag coefficient is weak. On the right panel of Fig.(4), the effect of $\xi / s$ and $\tau$ on the drag coefficient is shown. As expected, with an increase in $\xi / s$, the drag coefficient increases as shown by a dashed black and the blue curve i.e., $\xi / s=0.015$ (dashed black lines) and $\xi / s=0.03$ (blue lines) for $\tau=0.3 \mathrm{fm}^{-1}$. Same as earlier, with an increase in $\tau$, the drag coefficient decreases.

Drag coefficient $A(\eta)$ normalized with $A(\eta=0)$ as a function of HQ momentum $p$ for $T=220 \mathrm{MeV}$ is shown in Fig. 5. Here, we have also shown the results for HQ momenta $p \sim M$. The extrapolated results for higher momenta will not be reliable as we have not taken contribution from gluon radiation. As may be observed from the left panel of the same figure, for finite $\eta / s$, the 

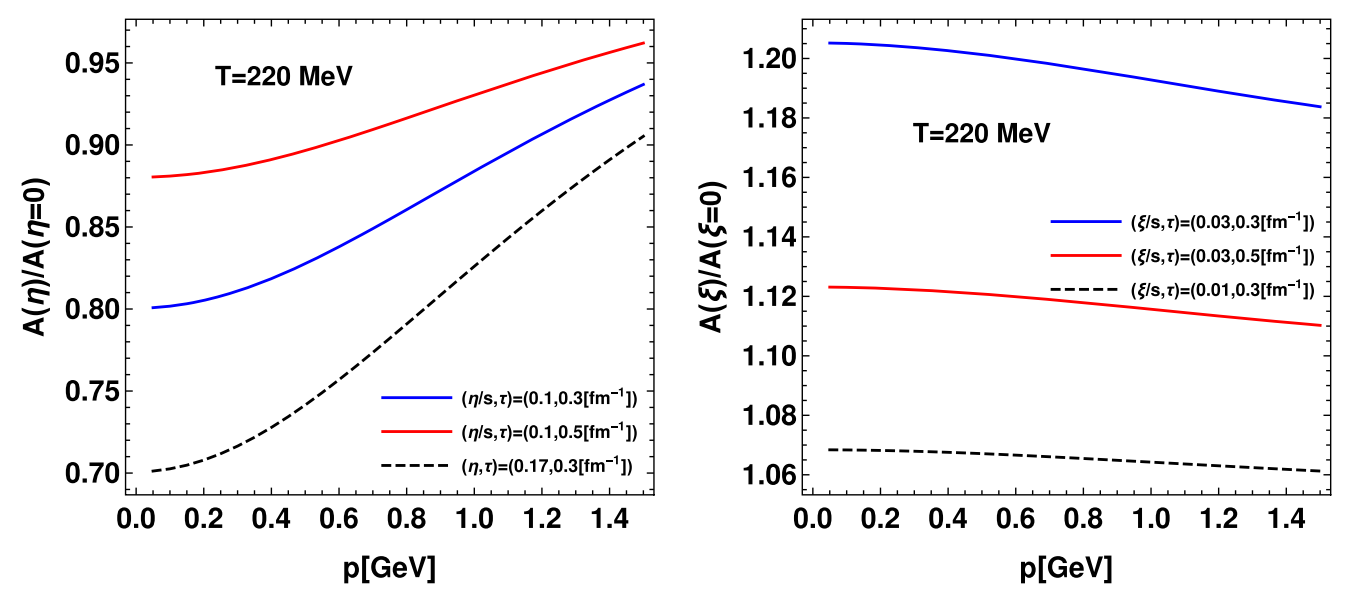

FIG. 5. Left panel: Variation of $A(\eta) / A(\eta=0)$ as a function of momentum for $\xi / s=0$ and $T=220 \mathrm{MeV}$. The red curve corresponds to $\eta / s=0.1, \tau=0.5 \mathrm{fm}^{-1}$, the blue curve corresponds to $\eta / s=0.1, \tau=0.3 \mathrm{fm}^{-1}$ and the dashed black curve corresponds to $\eta / s=0.17, \tau=0.3 \mathrm{fm}^{-1}$. Right panel: Variation of $A(\xi) / A(\xi=0)$ as a function of momentum for $\eta / s=0$ and $T=220 \mathrm{MeV}$. The blue curve corresponds to $\xi / s=0.03, \tau=0.3 \mathrm{fm}^{-1}$, the red curve corresponds to $\xi / s=0.03, \tau=0.5 \mathrm{fm}^{-1}$ and the dashed black curve corresponds to $\xi / s=0.01, \tau=0.3 \mathrm{fm}^{-1}$.

drag coefficient increases with an increase in the HQ momentum. However, with an increase in $\eta / s$, the drag coefficient decreases as shown by the black dashed curve $(\eta / s=0.17)$ and the blue curve $(\eta / s=0.1)$. As earlier, this behavior can be explained by taking account of phase space suppression (momentum dependent term in $\delta f_{a / a b}$ ). Same as earlier, with an increase in $\tau$, the drag coefficient decreases. On the right panel of the same figure, the effect of bulk viscosity on the HQ drag coefficient is shown. Here, the drag coefficient decreases with an increases in the HQ momentum. Note that unlike $\eta / s$, with increase in $\xi / s$, the drag coefficient increases. Same as earlier, with an increases in $\tau$ the drag coefficient decreases.

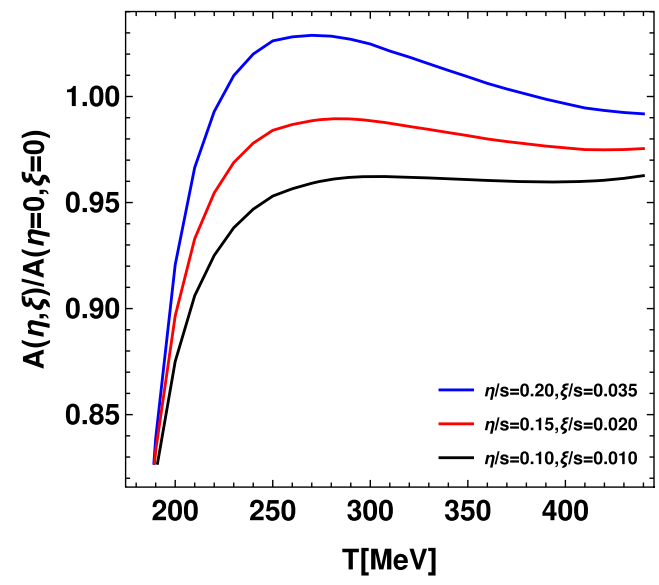

On the left panel of Fig. 6, the effect of both the bulk viscosity $(\xi / s)$ and the shear viscosity $(\eta / s)$ for $\tau=$ $0.3 \mathrm{fm}^{-1}$ on the normalized drag coefficient $(A(\eta, \xi) /$ $A(\eta=0, \xi=0))$ as a function of temperature is shown. At low temperature, shear viscosity dominates due to the phase space suppression so the drag coefficient decreases. At moderate temperature, i.e., around $250 \mathrm{MeV}$, the bulk viscosity dominates so the drag coefficient increases, again at high temperature, i.e., around $320 \mathrm{MeV}$, as seen earlier in Fig. 4, both $\eta / s$ and $\xi / s$ decreases the drag coefficient. As can be noted, for a smaller value of $\eta / s$ and $\xi / s$ e.g., $\eta / s=0.1, \xi / s=0.01$, the dependence of the drag coefficient on temperature is somewhat weak, however, the

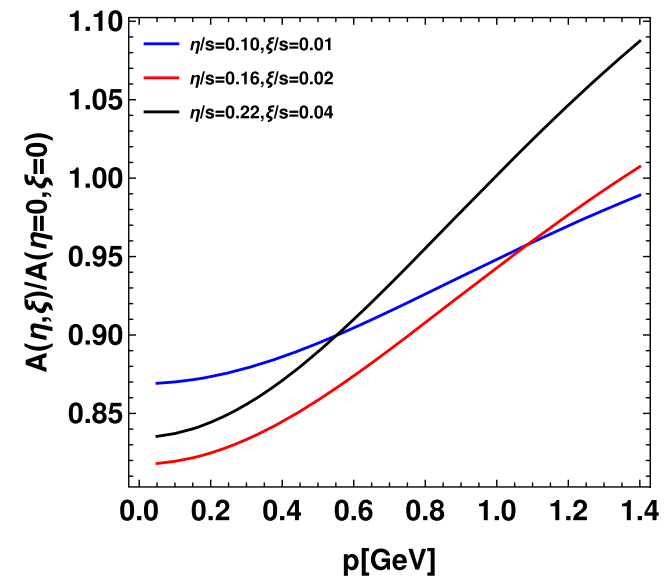

FIG. 6. Left panel: The ratio $A(\eta, \xi) / A(\eta=0, \xi=0)$ as a function of temperature for HQ momentum $p=1 \mathrm{GeV}^{\mathrm{and}} \tau=0.3$ fm ${ }^{-1}$. The blue curve corresponds to $\eta / s=0.2, \xi / s=0.035$, the red curve corresponds to $\eta / s=0.15, \xi / s=0.020$, and the black curve corresponds to $\eta / s=0.1, \xi / s=0.01$. Right panel: The ratio $A(\eta, \xi) / A(\eta=0, \xi=0)$ as a function of heavy quark momentum for $T=220 \mathrm{MeV}$ and $\tau=0.3 \mathrm{fm}^{-1}$. The blue curve corresponds to $\eta / s=0.1, \xi / s=0.01$, the red curve corresponds to $\eta / s=0.15$, $\xi / s=0.020$, and the black curve corresponds to $\eta / s=0.22, \xi / s=0.04$. 

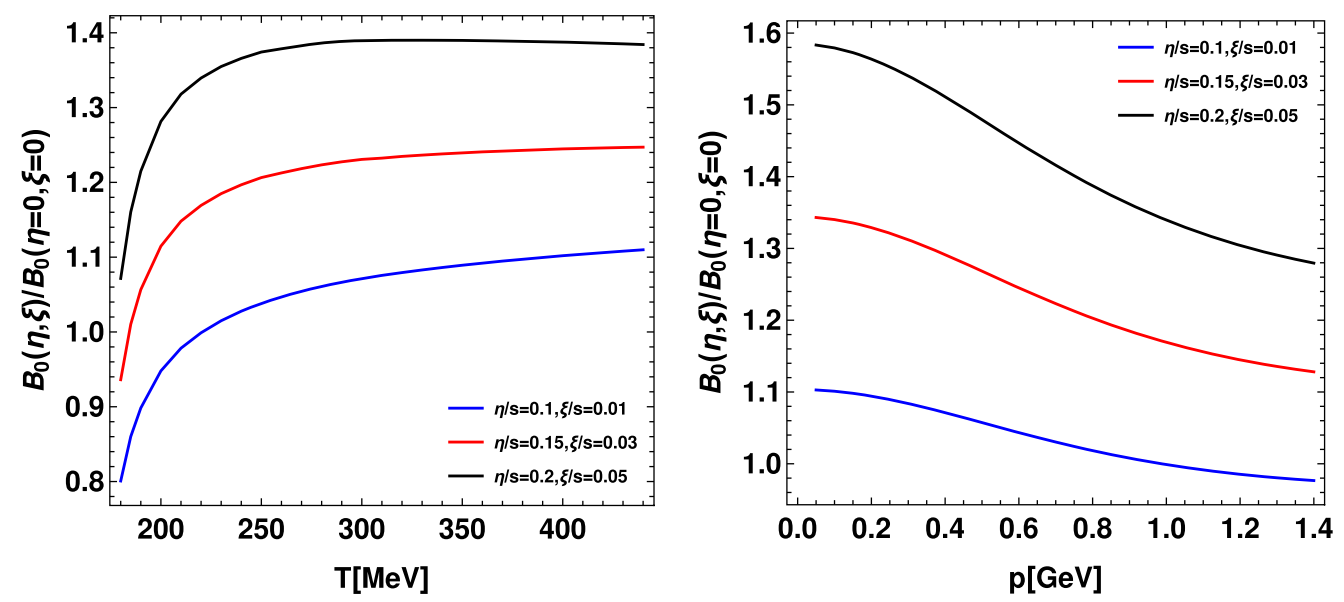

FIG. 7. Left panel: The ratio $B_{0}(\eta, \xi) / B_{0}(\eta=0, \xi=0)$ of diffusion coefficient as a function of temperature for HQ momentum $p=1 \mathrm{GeV}$ and $\tau=0.3 \mathrm{fm}^{-1}$. The black curve corresponds to $\eta / s=0.1, \xi / s=0.01$, the red curve corresponds to $\eta / s=0.15$, $\xi / s=0.03$, and the blue curve corresponds to $\eta / s=0.2, \xi / s=0.05$. Right panel: The ratio $B_{0}(\eta, \xi) / B_{0}(\eta=0, \xi=0)$ of diffusion coefficient as a function of HQ momentum for temperature $T=220 \mathrm{MeV}$ and $\tau=0.3 \mathrm{fm}^{-1}$. The black curve corresponds to $\eta / s=0.1$, $\xi / s=0.01$, the red curve corresponds to $\eta / s=0.15, \xi / s=0.03$, and the blue curve corresponds to $\eta / s=0.2, \xi / s=0.05$.

dependence is strong for a larger value of $\eta / s$ and $\xi / s$. On the right panel of Fig. 6 the same ratio as a function of momentum is plotted. Similar to the case of temperature behavior, for smaller values of $\eta / s$ and $\xi / s$ the drag coefficient is somewhat weakly dependent on the HQ momentum (see blue curve; $\eta / s=0.1, \xi / s=0.01$ ), however, it strongly depends on the same for larger values $\eta / s$ and $\xi / s$. Also note that at low momentum, for finite value of $\eta / s$ and $\xi / s$, the drag coefficient is small and increases with an increase in the HQ momentum.

The ratio $B_{0}(\eta, \xi) / B_{0}(\eta=0, \xi=0)$ of diffusion coefficients as defined in Eq. (16) is plotted as a function of temperature and momentum in Fig. 7. On the left panel of Fig. 7, the black curve corresponds to $\eta / s=0.2$, $\xi / s=0.05$, the red curve corresponds to $\eta / s=0.15$, $\xi / s=0.03$ and the blue curve corresponds to $\eta / s=0.1$, $\xi / s=0.01$. Here, we have taken $\tau=0.3 \mathrm{fm}^{-1}$ and the HQ momentum $p=1 \mathrm{GeV}$. It is observed that with an increase in $\eta / s, \xi / s$ and temperature, the diffusion coefficient increases. However, for smaller values of $\eta / s$ and $\xi / s$ e.g., $\eta / s=0.1, \xi / s=0.01$, the diffusion coefficient is not affected much. Note also that at low temperature for a smaller value of $\eta / s$ and $\xi / s$, e.g., blue curve, the diffusion coefficient is smaller as compared to the case of $\eta / s=0$, $\xi / s=0$. Similarly, as can be seen in the right panel of the same figure, with as increase in the HQ momentum the diffusion coefficient decreases. For the HQ momentum $p \ll M$, with an increase in $\eta / s$ and $\xi / s$, the diffusion coefficient increases. In pQCD, the results for the drag and the diffusion coefficients for various values of $\eta / s, \xi / s, \tau$ as a function of temperature and momentum that are presented here are similar as pointed out in Ref. [41]. However, the differences are due to the effect of the Polyakov loop.

It may be noted that with the Fokker-Planck formalism, one can relate the momentum diffusion coefficient $B_{0}(p)$ as estimated here to the spatial diffusion coefficient $D_{x}$ that appears, e.g., in the Ficks diffusion law. The diffusion coefficient $D_{x}$ is also estimated in the lattice QCD simulation. The two coefficients are related as [56]

$$
D_{x}=\frac{T^{2}}{B_{0}(p \rightarrow 0)} .
$$

In Fig. 8, we have plotted the quantity $2 \pi D_{x} T$ from leading order (LO) pQCD along with the lattice simulations and

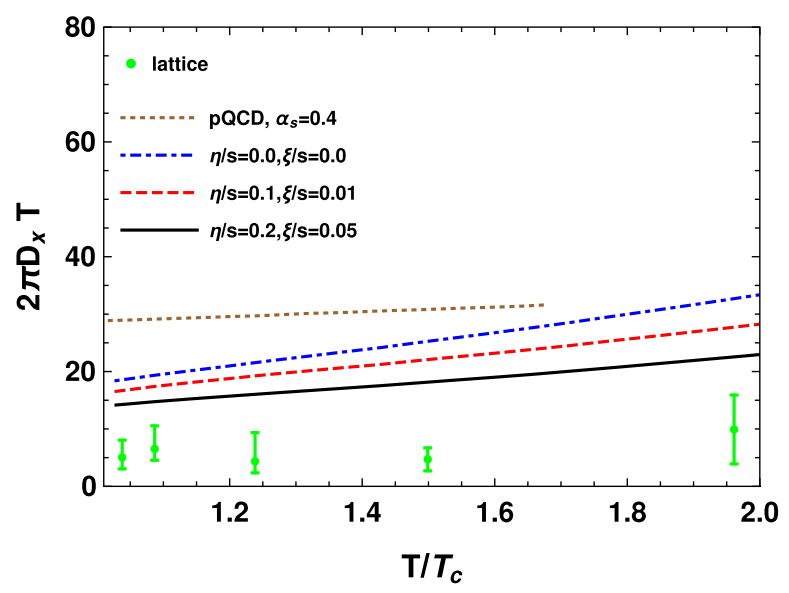

FIG. 8. The spatial diffusion $2 \pi D_{x} T$ as a function of temperature scaled by $T_{c}$ and $\tau=0.3 \mathrm{fm}^{-1}$. The blue curve (dashed dotted) corresponds to $\eta / s=0.0, \xi / s=0.0$, the red curve (dashed) corresponds to $\eta / s=0.1, \xi / s=0.01$, and the black curve (solid) corresponds to $\eta / s=0.2, \xi / s=0.04$. Here, the lattice data for Polyakov loop is taken from Ref. [44] and the QCD running coupling constant $\alpha_{s}$ as defined in Eq. (60). The brown curve (dotted) are LO pQCD results for constant coupling $\alpha_{s}=0.4$ [43] and the green (dots) are lattice results taken from the Ref. [18]. 
within the matrix model for various values of $\eta / s, \xi / s$ and $\tau=0.3 \mathrm{fm}^{-1}$ as a function of $T / T_{c}$. The brown dotted line is LO pQCD result for constant coupling $\alpha_{s}=0.4$. The blue (dashed dotted), red (dashed) and black (solid) lines are within the matrix model respectively for $\eta / s=0,0.1$, 0.2 and $\xi / s=0,0.01,0.05$. The green dots are the lattice results from Ref. [18]. The main observations in this figure are the following. The spatial diffusion coefficient is smaller compared to the perturbative QCD estimate. Inclusion of the viscous effect makes the coefficient even smaller. However, even with the inclusion of viscous effects as well as Polyakov loop, the spatial diffusion coefficient is still larger being almost about three times the corresponding lattice estimate. This indicates that there could be other nonperturbative effects possibly the contribution of finite light quark mass and also the radiative corrections for the estimation of diffusion coefficients.

\section{SUMMARY}

In the present work, we have computed the corrections due to the effects of the shear and the bulk viscosities on the HQ drag and diffusion coefficients within the matrix model of semi-QGP. To incorporate the viscous corrections we first write the distribution function of quark and gluon $\left(f_{a / c d}=f_{a / c d}^{0}+\delta f_{a / c d}\right.$, where $f_{a / c d}^{0}$ is equilibrium distribution function and $\delta f_{a / c d}$ summarizes the effect of shear and bulk viscosities) as defined in Eqs. (43) and (44). We next calculate the color dependent scattering amplitudes of
HQ from the light thermal partons in the bulk medium within the matrix model of semi QGP. Nonperturbative effects are included via the Polyakov loop in quark/ antiquark and gluon distribution functions as well as in the Debye mass. In all the calculations, we have taken the constant values for the viscosity to entropy density ratio, i.e., without their temperature dependence. With a reasonable constant value of $\eta / s$ for the temperature range we have considered, we find that the drag coefficient within the matrix model is small compared to that of perturbative QCD. Similarly, for a constant value of $\xi / s$, the drag coefficients is large within the matrix model compared the pQCD results. Furthermore, with an increase in temperature and momentum the drag coefficient increases, however, the diffusion coefficient increases with an increase in temperature and decreases with an increase in momentum. The spatial diffusion coefficient decreases with increase in the $\eta / s$ and $\xi / s$. For a small value of $\eta / s$ and $\xi / s$, both the drag and the diffusion coefficients have a weak dependence on temperature and momentum for all range of temperature and momentum considered here.

\section{APPENDIX: INTERFERENCE TERMS IN THE SCATTERING AMPLITUDE}

In this section, we shall discuss the interference amplitudes of $s, t$, and $u$ channel scatterings contributing to Compton scattering. For $s$ and $u$ channel of the scatterings, the interference term can be written as

$\mathcal{M}_{s} \mathcal{M}_{u}^{\dagger}=g^{4}\left(t^{e f}\right)_{a c}\left(t^{g h}\right)_{c b}\left(t^{g h}\right)_{a c^{\prime}}\left(t^{e f}\right)_{c^{\prime} b}\left[\frac{\left.\operatorname{Tr}\left[\left(\not p^{\prime}+M\right) \gamma^{\nu}(\not p+\not q+M) \gamma^{\mu}(\not p+M) \gamma^{\alpha}(\not p-\not)^{\prime}+M\right) \gamma^{\beta}\right] \epsilon_{\mu}(q) \epsilon_{\beta}(q) \epsilon_{\nu}^{*}\left(q^{\prime}\right) \epsilon_{\alpha}^{*}\left(q^{\prime}\right)}{\left(s-M^{2}\right)\left(u-M^{2}\right)}\right]$

where the trace is over Dirac matrices and the terms like $\left(t^{a b}\right)_{c d}$ are generators of the group which can be written in terms of projection operators as given in Eq. (19). Using the polarization sum for massless gluons, and spin sum/average of final/ initial quark, one can simplify Eq. (A1) to yield

$$
\mathcal{M}_{s} \mathcal{M}_{u}^{\dagger}=\frac{g^{4}}{16 N_{c}\left(N_{c}^{2}-1\right)} \mathcal{P}_{a c}^{e f} \mathcal{P}_{c b}^{g h} \mathcal{P}_{a c^{\prime}}^{g h} \mathcal{P}_{c^{\prime} b}^{e f}\left(\frac{-8 M^{2}\left(t-4 M^{2}\right)}{\left(s-M^{2}\right)\left(u-M^{2}\right)}\right)
$$

As earlier, color index $e f / g h$ of incoming/outgoing gluon will be summed with the statistical distribution function while evaluating the drag and the diffusion coefficients using Eq. (14). Similarly, another term that contributes to the amplitude, i.e., $\mathcal{M}_{s}^{\dagger} \mathcal{M}_{u}$ of the interference of the same scattering channels is given as

$$
\mathcal{M}_{s}^{\dagger} \mathcal{M}_{u}=\frac{g^{4}}{16 N_{c}\left(N_{c}^{2}-1\right)} \mathcal{P}_{a c}^{e f} \mathcal{P}_{c b}^{g h} \mathcal{P}_{a c^{\prime}}^{g h} \mathcal{P}_{c^{\prime} b}^{e f}\left(\frac{-8 M^{2}\left(t-4 M^{2}\right)}{\left(s-M^{2}\right)\left(u-M^{2}\right)}\right)
$$

For $s$ and $t$ channel scatterings, the matrix element squared of the interference term can be given as

$$
\mathcal{M}_{s} \mathcal{M}_{t}^{\dagger}=g^{4}\left(t^{e f}\right)_{a c}\left(t^{g h}\right)_{c b}\left(t^{l m}\right)_{b a} f^{d c, f e, h g}\left[\frac{\left.\operatorname{Tr}\left[(\not)^{\prime}+M\right) \gamma^{\nu}(\not p+\not t+M) \gamma^{\mu}(\not p+M) \gamma^{\lambda} C^{\alpha \beta \sigma}\left(q-q^{\prime},-p, p^{\prime}\right) g_{\alpha \beta}\right] \epsilon_{\mu} \epsilon_{\alpha} \epsilon_{\nu}^{*} \epsilon_{\sigma}^{*}}{\left(s-M^{2}\right)\left(t+\left(m_{D}^{2}\right)_{m l c d}\right)}\right],
$$


where $C^{\mu \nu \sigma}$ is defined in Eq. (58). With the polarization sum of massless gluon and trace over Dirac space, the scattering amplitude can be simplified to

$$
\mathcal{M}_{s} \mathcal{M}_{t}^{\dagger}=\mathcal{M}_{s}^{\dagger} \mathcal{M}_{t}=\frac{g^{4} \sqrt{2}}{16 N_{c}\left(N_{c}^{2}-1\right)} \mathcal{P}_{a c}^{e f} \mathcal{P}_{c b}^{g h} \mathcal{P}_{b a}^{l m} f^{d c, f e, h g}\left(\frac{16 M^{2} s-8 M^{4}-u s}{\left(s-M^{2}\right)\left(t+\left(m_{D}^{2}\right)_{m l c d}\right)}\right)
$$

The last term contributing to the scattering amplitude of the interfering diagrams comes from the $u$ and $s$ channel scatterings and can be given as

$\mathcal{M}_{u} \mathcal{M}_{t}^{\dagger}=g^{4}\left(t^{g h}\right)_{a c}\left(t^{e f}\right)_{c b}\left(t^{l m}\right)_{b a} f d c, f e, h g\left[\frac{\operatorname{Tr}\left[(\not p+M) \gamma^{\mu}\left(\not p^{\prime}-\not q+M\right) \gamma^{\nu}(\not p+M) \gamma^{\lambda} C^{\alpha \beta \sigma}\left(q-q^{\prime},-q,-q^{\prime}\right) g_{\lambda \alpha}\right] \epsilon_{\mu} \epsilon_{\alpha} \epsilon_{\nu}^{*} \epsilon_{\sigma}^{*}}{\left(u-M^{2}\right)\left(t+\left(m_{D}^{2}\right)_{m l c d}\right)}\right]$.

Similar to Eqs. (A1) and (A4), Eq. (A6) can be simplified to

$$
\mathcal{M}_{u} \mathcal{M}_{t}^{\dagger}=\mathcal{M}_{u}^{\dagger} \mathcal{M}_{t}=\frac{g^{4} \sqrt{2}}{16 N_{c}\left(N_{c}^{2}-1\right)} \mathcal{P}_{b c}^{g h} \mathcal{P}_{c a}^{e f} \mathcal{P}_{a b}^{l m} f^{d c, f e, h g}\left(\frac{8\left(M^{4}-2 M^{2} u+u s\right)}{\left(u-M^{2}\right)\left(\left(t+\left(m_{D}^{2}\right)_{m l c d}\right)\right)}\right)
$$

[1] S. S. Adler et al. (PHENIX Collaboration), Phys. Rev. Lett. 91, 182301 (2003).

[2] C. Adler et al. (STAR Collaboration), Phys. Rev. Lett. 90, 032301 (2003).

[3] M. Aaboud et al. (ATLAS Collaboration), Phys. Rev. C 98, 044905 (2018).

[4] A. Adare et al. (PHENIX Collaboration), Phys. Rev. Lett. 98, 172301 (2007).

[5] X. Dong, arXiv:1810.00996.

[6] R. Rapp et al., Nucl. Phys. A979, 21 (2018).

[7] B. I. Abelev et al. (STAR Collaboration), Phys. Rev. Lett. 98, 192301 (2007); 106, 159902(E) (2011).

[8] H. Satz, Lect. Notes Phys. 945, 1 (2018).

[9] M. Cacciari, M. Greco, and P. Nason, J. High Energy Phys. 05 (1998) 007.

[10] M. Cacciari, S. Frixione, N. Houdeau, M. L. Mangano, P. Nason, and G. Ridolfi, J. High Energy Phys. 10 (2012) 137.

[11] S. Wicks, W. Horowitz, M. Djordjevic, and M. Gyulassy, Nucl. Phys. A784, 426 (2007).

[12] M. Mannarelli and R. Rapp, Phys. Rev. C 72, 064905 (2005).

[13] S. Y. F. Liu and R. Rapp, Phys. Rev. C 97, 034918 (2018).

[14] T. Song, H. Berrehrah, D. Cabrera, J. M. Torres-Rincon, L. Tolos, W. Cassing, and E. Bratkovskaya, Phys. Rev. C 92, 014910 (2015).

[15] S. K. Das, F. Scardina, S. Plumari, and V. Greco, Phys. Lett. B 747, 260 (2015).

[16] H. Berrehrah, E. Bratkovskaya, W. Cassing, P. B. Gossiaux, J. Aichelin, and M. Bleicher, Phys. Rev. C 89, 054901 (2014).

[17] H. van Hees and R. Rapp, Phys. Rev. C 71, 034907 (2005).
[18] D. Banerjee, S. Datta, R. Gavai, and P. Majumdar, Phys. Rev. D 85, 014510 (2012).

[19] H. T. Ding, A. Francis, O. Kaczmarek, F. Karsch, H. Satz, and W. Soeldner, Phys. Rev. D 86, 014509 (2012).

[20] A. Francis, O. Kaczmarek, M. Laine, T. Neuhaus, and H. Ohno, Phys. Rev. D 92, 116003 (2015).

[21] H. van Hees, M. Mannarelli, V. Greco, and R. Rapp, Phys. Rev. Lett. 100, 192301 (2008).

[22] B. Singh, A. Abhishek, S. K. Das, and H. Mishra, Phys. Rev. D 100, 114019 (2019).

[23] Z. Qiu, C. Shen, and U. Heinz, Phys. Lett. B 707, 151 (2012).

[24] P. Romatschke and U. Romatschke, Phys. Rev. Lett. 99, 172301 (2007).

[25] R. S. Bhalerao, A. Jaiswal, and S. Pal, Phys. Rev. C 92 , 014903 (2015).

[26] B. Schenke, S. Jeon, and C. Gale, Phys. Rev. C 85, 024901 (2012).

[27] M. Prakash, M. Prakash, R. Venugopalan, and G. Welke, Phys. Rep. 227, 321 (1993).

[28] G. Baym, H. Monien, C. J. Pethick, and D. G. Ravenhall, Phys. Rev. Lett. 64, 1867 (1990).

[29] P. B. Arnold, G. D. Moore, and L. G. Yaffe, J. High Energy Phys. 05 (2003) 051.

[30] D. Kharzeev and K. Tuchin, J. High Energy Phys. 09 (2008) 093.

[31] K. Dusling and T. Schfer, Phys. Rev. C 85, 044909 (2012).

[32] G. Vujanovic, C. Young, B. Schenke, R. Rapp, S. Jeon, and C. Gale, Phys. Rev. C 89, 034904 (2014).

[33] J. R. Bhatt, H. Mishra, and V. Sreekanth, Nucl. Phys. A875, 181 (2012). 
[34] J. R. Bhatt, H. Mishra, and V. Sreekanth, J. High Energy Phys. 11 (2010) 106.

[35] S. Sarkar and A. K. Dutt-Mazumder, Phys. Rev. D 88, 054006 (2013).

[36] S. Sarkar, C. Chattopadhyay, and S. Pal, Phys. Rev. C 97, 064916 (2018).

[37] R. Baier, A. H. Mueller, and D. Schiff, Phys. Lett. B 649, 147 (2007).

[38] R. Baier, D. Schiff, and B. G. Zakharov, Annu. Rev. Nucl. Part. Sci. 50, 37 (2000).

[39] C. Chattopadhyay, R. S. Bhalerao, J. Y. Ollitrault, and S. Pal, Phys. Rev. C 97, 034915 (2018).

[40] Z. Qiu and U. Heinz, Phys. Lett. B 717, 261 (2012).

[41] S. K. Das, V. Chandra, and J. e. Alam, J. Phys. G 41, 015102 (2013).

[42] B. Svetitsky, Phys. Rev. D 37, 2484 (1988).

[43] R. Rapp and H. van Hees, arXiv:0903.1096.

[44] A. Bazavov, N. Brambilla, H.-T. Ding, P. Petreczky, H.-P. Schadler, A. Vairo, and J.H. Weber, Phys. Rev. D 93, 114502 (2016).
[45] R. D. Pisarski and V. V. Skokov, Phys. Rev. D 94, 034015 (2016).

[46] Y. Hidaka and R. D. Pisarski, Phys. Rev. D 80, 036004 (2009).

[47] G. 't Hooft, Nucl. Phys. B72, 461 (1974).

[48] P. Cvitanovic, Phys. Rev. D 14, 1536 (1976).

[49] Y. Hidaka and R. D. Pisarski, Phys. Rev. D 81, 076002 (2010).

[50] G. D. Moore and D. Teaney, Phys. Rev. C 71, 064904 (2005).

[51] D. Teaney, Phys. Rev. C 68, 034913 (2003).

[52] K. Dusling and D. Teaney, Phys. Rev. C 77, 034905 (2008).

[53] K. Dusling, G. D. Moore, and D. Teaney, Phys. Rev. C 81, 034907 (2010).

[54] J. D. Bjorken, Phys. Rev. D 27, 140 (1983).

[55] W. E. Caswell, Phys. Rev. Lett. 33, 244 (1974).

[56] L. M. Abreu, D. Cabrera, F. J. Llanes-Estrada, and J. M. Torres-Rincon, Ann. Phys. (Amsterdam) 326, 2737 (2011). 